
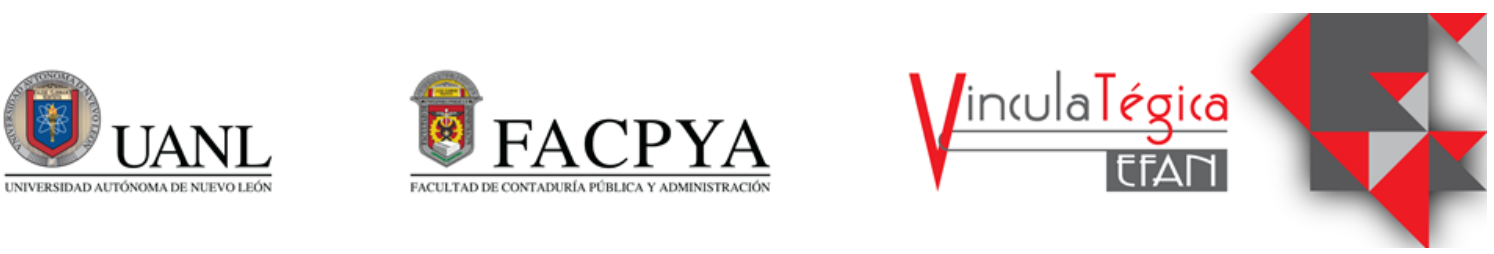

\title{
El análisis factorial confirmatorio aplicado a constructos del sector comercial en Azogues (Ecuador)
}

\author{
Juan Solis Muñoz ${ }^{1}$;énesis Toledo Mora ${ }^{2}$ y Dayana Urgilez Idrovo ${ }^{3}$ \\ ${ }^{1}$ Universidad Católica de Cuenca. Unidad Académica de Administración. Carrera de Administración de \\ Empresas. Departamento de Investigación. Avenida Ernesto Che Guevara y Avenida 16 de abril, Azogues, \\ Cañar, Ecuador.jbsolizm@ucacue.edu.ec, (07) 2240901. \\ ${ }^{2}$ Universidad Católica de Cuenca Sede Azogues. Unidad Académica de Administración. Carrera de \\ Administración de Empresas. genesis.toledo@est.ucacue.edu.ec \\ ${ }^{2}$ Universidad Católica de Cuenca Sede Azogues. Unidad Académica de Administración. Carrera de \\ Administración de Empresas.jdurgilezi43@est.ucacue.edu.ec \\ Información del artículo revisado por pares \\ Fecha de aceptación: junio-2021 \\ Fecha de publicación en línea: diciembre-2021 \\ DOI: https://doi.org/10.29105/vtga7.2-50
}

\section{Resumen}

El presente artículo parte de la necesidad de contar con una herramienta validada para el estudio de las MiPymes en el sector comercial del cantón Azogues, Ecuador. Se trata de un estudio cuantitativo a una población de 160 emprendimientos dedicados al comercio. Los sujetos de investigación son los emprendedores asociados en la Cámara de Comercio e Industrias de Azogues. La encuesta se hace a un nivel de confianza del $95 \%$ con un límite de error de estimación del 5\%. El diseño del instrumento se sujetó a validación por consenso de expertos respetando el perfil requerido. Los constructos teóricos se basan en los hallazgos identificados en la revisión sistemática de la literatura alrededor del emprendimiento y son: Valor para el cliente, transformación digital, ventaja competitiva y Liderazgo. Se parte de 45 items originales y con la aplicación del Análisis Factorial Confirmatorio se reduce a 17 items para los 4 constructos con una carga factorial igual o superior a 0.6.

Palabras clave: Sector comercial, Análisis Factorial Confirmatorio, Emprendimiento, Ecuador.

\begin{abstract}
:
This article starts from the need to have a validated tool for the study of Mipymes in the commercial sector of the canton Azogues, Ecuador. This is a quantitative study of a population of 160 businesses dedicated to commerce. The research subjects are the entrepreneurs associated with the Chamber of Commerce and Industries of Azogues. The survey is done at a confidence level of $95 \%$ with a limit of error of estimation of $5 \%$. The design of the instrument was subjected to validation by expert consensus, respecting the required profile. The theoretical constructs are based on the findings identified in the systematic review of the literature around entrepreneurship and are: Value for the client, digital transformation, competitive advantage and Leadership. It starts with 45 original items and with the application of the Confirmatory Factor Analysis it is reduced to 17 items for the 4 constructs with a factor load equal to or greater than 0.6.
\end{abstract}

Keywords: Commercial sector, Confirmatory Factor Analysis, Entrepreneurship, Ecuador. 


\section{INTRODUCCIÓN}

Según un informe de la Cámara de Comercio de Guayaquil (2021) en el contexto de la reforma laboral los sectores que más empleo han generado en los 5 últimos años en el Ecuador es la agricultura que concentró el $30 \%$ de los empleos seguido por el comercio, $17,5 \%$ y la manufactura con el $10,5 \%$. Cabe indicar que el sector que mayor cantidad de empleo adecuado generó es el comercio con el 16,4\%, le sigue la enseñanza, $13,6 \%$ y la manufactura con el $12,9 \%$.

Ahora bien, la pandemia del Covid-19 según cifras del Banco Central del Ecuador decreció la economía en un 12,4\% en el segundo semestre del año 2020 afectando principalmente a la agricultura que presentó un decrecimiento interanual del 1,2\% y al Valor Agregado Bruto del comercio que registró una variación negativa de 9,8\% (Banco Central del Ecuador, 2021).

Un estudio del Grupo de Investigación en Economía Regional (2020) da cuenta que las ventas durante el primer semestre del año 2020 en Cuenca, provincia del Azuay cayeron en 2 mil 100 millones de USD. La investigación revela que la reducción en las ventas se aproximó al $70 \%$ porque muchas empresas tuvieron una paralización total.

En el cantón Azogues, provincia del Cañar en el escenario pre pandemia el comercio y la construcción se consolidaron. Según el Banco Central del Ecuador (2018) el Cañar recibió 2 mil 033 millones de USD por remesas entre el 2008 y el 2017 y aunque no existen por ahora estudios que midan el impacto del Covid-
19 en estos sectores, se hace necesario contar con constructos teóricos y herramientas de medición validados para las MiPymes del sector comercial del cantón Azogues.

\section{MARCO TEÓRICO}

En este apartado se describen los aportes teóricos del Análisis Factorial Confirmatorio y las variables de los constructos teóricos que más se aproximan a la necesidad de contar con una herramienta para analizar los datos.

\subsection{Análisis Factorial Confirmatorio}

En los últimos años el Análisis Factorial Confirmatorio (AFC), se ha vuelto en una de las herramientas de análisis más empleadas en indagaciones de ciencias sociales, esto debido a la facilidad que brinda al momento de trabajar con datos reducidos, donde las variables son más fáciles de manipular con el fin de evitar errores al momento de avaluar y analizar datos, de esta manera, facilita al investigador justificar los errores de medición entre las variables (Arauz, 2015).

El AFC es un proceso de investigación contenido en los modelos de ecuaciones estructurales, teniendo como objetivo el análisis de los modelos de medida, es decir, se centra en examinar las relaciones existentes entre un grupo de variables observadas y variables latentes. De esta forma, las variables observadas pueden corresponder a ítems o preguntas de un test o resultados obtenidos de la aplicación de herramientas de categorización conductual (Arias, 2008). El Análisis Factorial Confirmatorio, es el más utilizado al momento 
de evidenciar la valides de un constructo, es decir, ayuda a obtener información sobre los instrumentos de medida, de manera que se pueda aceptar o rechazar ciertas hipótesis (Morata, Holgado, Barbero y Méndez, 2015).

En la línea de pensamiento de Luna y Laca (2014), el análisis factorial confirmatorio consiste en resumir la información contenida de una matriz de datos $\mathrm{y}$, posteriormente, establecer un modelo donde se construye una hipótesis previa de las variables, debido a esto el análisis factorial confirmatorio suele ser considerado como una técnica de estudio que es aplicada para conocer estándares que demuestren una confiabilidad adecuada. En el análisis factorial confirmatorio, el modelo de ajuste proporciona una orientación hacia el nivel de compatibilidad que se presenta en la medición de los datos al momento de realizar una corrección estandarizada, y su evaluación es realizada con diferentes indicadores o índices de ajustes, los mismo que pueden ser denominados como métodos absolutos o comparativos (Pérez, 2020).

Cabe destacar que, tal como escribió Arias (2008), una de las principales características con las que cuenta el AFC, es que la persona que realiza la investigación debe sintetizar y especificar los elementos principales del modelo de investigación diseñado, es decir, los factores e indicadores presentados, los mismos que deben fundamentarse en bases teóricas con el fin de comprobar teorías e hipótesis. De la misma manera, Arauz (2015), afirma que el análisis factorial confirmatorio, es un instrumento que se usa para evaluar un modelo de medición fundamentado en bases teóricas o empíricas que requieren ser comprobadas. Además, recalca que el investigador también puede presentar las cargas factoriales con las que cuenta cada factor.

En el análisis factorial confirmatorio, la valoración que cada persona establece en relación con los ítems que se presentan, se genera por una variable no observada, que es conocida y denominada como factor latente, la cual expone la variabilidad de cada uno de los ítems. Sin embargo, no se puede revelar de manera total la variabilidad de las respuestas, por lo que a este segmento no explicado se le llama error de media. Además, se considera necesario que el modelo diseñado exponga una buena relación de datos y, a la vez, la dirección y trascendencia de los parámetros que lo componen (Herrero, 2010).

Para Domínguez (2019), al momento de la revisión de un nuevo modelo del AFC se exhiben las cargas factoriales que presenta cada ítem y se brinda la información sobre el nivel de error de cada uno, el cual también es conocido como valor residual, esto varía dependiendo de las preguntas planteadas, es decir, con diferentes aspectos, tales como: estado o rasgo de una persona con la condición física y así con un sinnúmero más de variables.

En las diferentes revisiones bibliográficas sobre el análisis factorial confirmatorio se puede observar que se da como continuidad del análisis factorial exploratorio para proceder de mejor forma a obtener resultados más óptimos (viables). En efecto, “el 
índice de ajuste para los modelos anidados depende en buena medida del modelo elegido como línea base de comparación, cuanto menos restrictivo sea el modelo nulo, mayor será el índice de ajuste obtenido" (De Frutos, Ruiz y San Martín, 1998, p. 353).

En el artículo titulado Análisis factorial confirmatorio de las dimensiones del compromiso con la organización se observa claramente, que utilizan diferentes tipos de programas tales son: Prelis, y Lisrel 7 que sirven para las diversas valoraciones que le corresponden a las matrices según cada caso de estudio factorial confirmatorio que se encuentre en desarrollo. De acuerdo con los razonamientos que se han venido realizando, los múltiples métodos, técnicas e instrumentos que se apliquen en los análisis factoriales ya sean estos exploratorios o confirmatorios necesitan valides de estadísticos y coeficientes como el Alfa de Cronbach para conseguir con cimientos cuantitativos un correcto estudio de investigación. Por las consideraciones anteriores, en el artículo "Análisis Factorial Confirmatorio para medir las limitantes percibidas en el pregrado para el desarrollo de actividades de investigación" se puede reflexionar y corroborar que guardan cierto grado de relación el análisis factorial exploratorio con el confirmatorio, por ejemplo, dicho estudio indica.

Como la estructura no reporta valores totalmente aceptables para este cálculo, se hace necesario corroborar el constructo teórico a través de otros métodos y es por ello que se realizó un análisis factorial confirmatorio, con el cual se confirmó la sustentabilidad empírica del modelo de medida propuesto, lo que se muestra en los valores de los principales indicadores de ajuste. (Magaña, Aguilar y Vázquez, 2017, p.526)

Lo expuesto se corrobora con el aporte de Zumbo (2007), en su opinión el análisis factorial confirmatorio es la técnica más utilizada para adquirir evidencias sobre la validez del constructo que estamos desarrollando, puesto que informa sobre la distribución interna de los materiales de medida. Cabe recalcar que para realizar este análisis factorial primeramente se tiene que especificar o identificar el modelo de investigación que estamos realizando. Tras la recogida de datos, se estiman los parámetros $\mathrm{y}$, a continuación, se evalúa el ajuste del modelo. $\mathrm{Si}$ el modelo presenta un ajuste adecuado, se podrá utilizar para evaluar e interpretar los parámetros.

En base a la investigación realizada (Sternberg, et al., 2000), menciona que una vez realizada la estrategia general se procede a comparar aquellos modelos añadidos jerárquicamente, luego de comparar los resultados de los diferentes modelos estructurales que corresponden a diferentes hipótesis. También adjunto a su comentario que los modelos se analizan en base al método de máxima verosimilitud, debido a que los valores de aquellos medios de los índices de asimetría y curtosis de los items caen dentro de un rango de valores establecido.

En el momento que se realizar un AFC, la persona que investiga tiene que basarse en la 
teoría de la misma para especificar el número de factores existentes para un conjunto de variables, seguidamente nos indica que el factor en el que se cargará cada variable. La finalidad de este método es examinar que tanto se ajusta la especificación teórica de los factores hacia la realidad de los resultados adquiridos en el estudio (Herrero, 2010).

\subsection{Variables de investigación}

En esta sección se estudian las variables que conforman los constructos teóricos alrededor del emprendimiento con enfoque en las MiPymes del sector comercial del cantón Azogues, Ecuador.

\subsubsection{Valor para el cliente}

Según un estudio realizado por Viscarri (2011) los emprendimientos que encaran la misión de generar valor para el cliente en un micro y macro entorno complejo donde el consumidor cada vez busca una propuesta de valor deben insertarse en las tendencias del mercado. Se trata de identificar con precisión las palancas clave del sector comercial y comunicar los beneficios del producto a los clientes.

El valor para el cliente se estudia como una propuesta de valor implica que los clientes reciban todos los beneficios de un bien o servicio que hace que lo perciban como la mejor alternativa. De esta forma los referidos se propagan con el máximo valor recibido en el presente y en el futuro (Anderson, Narus \& Van Rossum, 2006).

\subsubsection{Transformación digital}

La transformación digital representa un nuevo modelo de organización y control de la cadena de valor mediante el ciclo de vida del producto de la mano de los procesos de fabricación con base en las tecnologías de información y comunicación (Suárez, Lloret y Mengual, 2016).

Un estudio en el contexto colombiano sobre el impacto de la transformación digital en la Universidad Nacional explica que la competitividad del emprendimiento de educación superior se ha tornando sostenible en el tiempo lo que implica una transformación cultural de los actores. La plataforma administrativa y académica se ha puesto a tono con las nuevas realidades tecnológicas del entorno (Serna, Benavides y Burgos, 2018).

\subsubsection{Ventaja competitiva}

Sobre las ventajas que genera el emprendimiento por ahora no existe una respuesta contundente más bien se admite que el emprendimiento es una actividad que desarrolla ventajas competitivas en sus actores. La ventaja competitiva se entiende como la estrategia de vanguardia que imponen los emprendimientos para generar valor a largo plazo (Peñaloza, 2019).

La ventaja competitiva se convierte en capacidades distintas de la gestión estratégica lo que genera una cultura organizacional orientada a la innovación que busca satisfacer continuamente las necesidades insatisfechas de los clientes (Valencia et al., 2017).

\subsubsection{Liderazgo}


Se encuentra que el Liderazgo en las actividades de emprendimiento es esencialmente importante porque garantiza que el emprendedor tenga mayores competencias en el momento de sostener la gestión del emprendimiento. Una revisión sistemática sobre el liderazgo y el emprendimiento concluye que el emprendedor debe ser autodidacta y debe ser un líder reconocido lo que le permitirá sacar del valle de la muerte al emprendimiento (Buitrago, 2020).

El liderazgo desde la perspectiva del emprendimiento sostenible encuentra que el líder debe convertirse en una persona con condiciones específicas que alienten un entorno favorable para el emprendimiento propiciando un clima y una cultura organizacional eficiente (Villamil, 2016).

\section{METODO}

En esta sección se desarrolla el método de estudio del presente artículo. Se trata del método científico aplicado al desarrollo del Análisis Factorial Confirmativo AFC.

\subsection{El problema de investigación}

Al momento de analizar el comportamiento de los emprendimientos del sector comercial de Azogues, Ecuador se torna imprescindible conocer las variables latentes que se corresponden con los constructos teóricos recomendados por el marco teórico para aislar las cargas factoriales irrelevantes.

\subsection{Pregunta central de investigación}

La pregunta central de investigación se plantea alrededor del problema de investigación: ¿Qué variables tienen la mayor carga factorial en el constructo teórico mediante la aplicación del Análisis Factorial Confirmatorio?

\subsection{Objetivo general de la investigación}

Confirmar las variables con mayor carga factorial mediante el Análisis Factorial Confirmatorio para validar el constructo a ser aplicado en el análisis de los datos del sector comercial de Azogues, Ecuador.

\subsection{Hipótesis general de investigación}

Las variables con mayor carga factorial que conforman el constructo teórico por componente rotados para analizar los datos del sector comercial de Azogues, Ecuador son: Valor para el cliente, transformación digital, ventaja competitiva y liderazgo.

\subsection{Justificación y aportaciones del estudio}

3.5.1 Práctica: La presente investigación ayudará a fundamentar los constructos para una mejor aplicación de estudios empíricos en el sector comercial del cantón Azogues, Ecuador de modo que el gerenciamiento de las MiPymes se base en evidencia científica demostrado en los constructos del Análisis Factorial Confirmatorio.

3.5.2 Teórica: Se justifica teóricamente el estudio porque se utilizan teorías y estudios aplicados alrededor del emprendimiento y los constructos de valor para el cliente, transformación digital, ventaja competitiva y liderazgo.

3.5.3 Metodológica: Esta investigación tiene una justificación metodológica porque se fundamenta en el método científico que permite determinar los constructos teóricos del 
emprendimiento de las MiPymes en el sector comercial de Azogues, Ecuador aplicando el Análisis Factorial Confirmatorio. Se parte de una exhaustiva revisión de la literatura que permite determinar a priori que valor para el cliente, transformación digital, ventaja competitiva y liderazgo son las variables que inciden en el emprendimiento. Para el estudio de campo se considera una población de 160 locales comerciales. Los sujetos de investigación con los comerciantes asociados a la Cámara de Comercio e Industrias de Azogues. Se determina una muestra de 157 elementos muestrales con un nivel de confianza del 95\% y un límite de error de estimación del 5\% con 2 desviaciones estándar. Se estructura el instrumento de medición con 45 items iniciales valido por consenso de jueces. Se aplica el instrumento a 101 sujetos de investigación. Se tiene como marco muestral el listado de emprendedores del sector comercial entregado por la Cámara de Comercio e Industrias de Azogues. Se utiliza el Software del SPSS para el Análisis Factorial Confirmatorio.

\subsection{Limitaciones del estudio}

El presente estudio se realizó en el contexto de la pandemia del Covid-19 lo que limitó a que el trabajo de campo se levante por llamadas telefónicas. Además, se trata de un estudio transversal y no experimental.

\section{RESULTADOS}

En esta sección se muestran los resultados del Análisis Factorial Confirmatorio. La literatura determina que el Emprendimiento representa a los constructos de Valor para el Cliente,
Transformación Digital, Ventaja Competitiva y Liderazgo. La prueba de KMO y Bartlett, medida Kaiser-Meyer-Olkin de adecuación de muestreo es de 0.828 con una Sig. 000 menor a 0.05 y por tanto estadísticamente significativa (véase la tabla 1).

\begin{tabular}{|l|r|r|}
\multicolumn{1}{|c|}{ Tabla 1 Matrices anti -imagen y comunalidades } \\
\hline Variables observables & $\begin{array}{c}\text { Correlación } \\
\text { anti-imagen }\end{array}$ & Comunalidades \\
\hline P6 Alternativa de negocio & $0.836 \mathrm{a}$ & 0.882 \\
\hline P7 Margen de mejora & $0.81 \mathrm{a}$ & 0.914 \\
\hline P8 Adaptación tecnológica & $0.885 \mathrm{a}$ & 0.857 \\
\hline P9 Tienda virtual & $0.864 \mathrm{a}$ & 0.751 \\
\hline P10 Impactos económicos & $0.92 \mathrm{a}$ & 0.821 \\
\hline P18 Orientación a consumidores & $0.654 \mathrm{a}$ & 0.707 \\
\hline P21 Adaptabilidad a cambios & $0.805 \mathrm{a}$ & 0.757 \\
\hline P22 Capacidad de resiliencia & $0.767 \mathrm{a}$ & 0.595 \\
\hline P25 Nuevas ideas & $0.841 \mathrm{a}$ & 0.654 \\
\hline P26 Creatividad & $0.829 \mathrm{a}$ & 0.647 \\
\hline P28 Invención & $0.816 \mathrm{a}$ & 0.724 \\
\hline P33 Integración de la ogranización & $0.815 \mathrm{a}$ & 0.713 \\
\hline P34 Capacidad de reinvención & $0.816 \mathrm{a}$ & 0.729 \\
\hline P36 Convicción de argumentos & $0.845 \mathrm{a}$ & 0.653 \\
\hline P38 Consecución de metas & $0.784 \mathrm{a}$ & 0.824 \\
\hline P39 Organización y coordinación & $0.832 \mathrm{a}$ & 0.766 \\
\hline P44 Cultura de innovación & $0.852 \mathrm{a}$ & 0.699 \\
\hline
\end{tabular}

Fuente: Elaboración propia

La Varianza total explicada muestra que los cuatro componentes explican el 75,37\% de la Varianza. Lo cual corrobora el gráfico de sedimentación que a partir el componente 4, el eje de las X se aplana (Véase figura 1).

Figura 1 Grafico de sedimentación

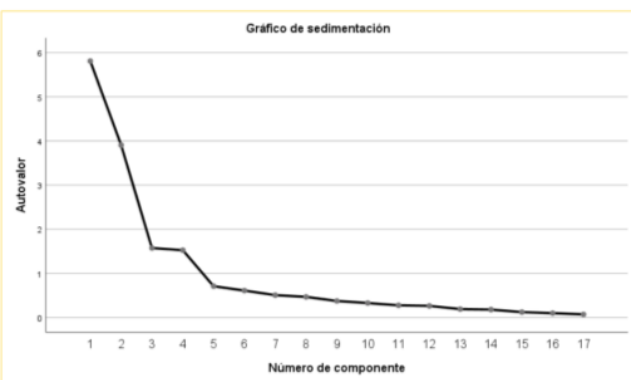

Fuente: Elaboración propia

Para explicar la matriz de componentes rotados se debe indicar que originalmente se partió de 45 items alrededor de las dimensiones 
valor para el cliente 5, transformación digital 7, ventaja competitiva 16, liderazgo 11 y emprendimiento 6. La matriz de componentes rotados presenta una carga factorial igual o mayor a 0.6 y agrupa a las variables observables alrededor de los cuatro constructos que la literatura aconseja (Veáse tabla 2).

Tabla 2 Matriz de componentes rotados

\begin{tabular}{|l|r|r|r|c|}
\hline & \multicolumn{4}{|c|}{ Componentes } \\
\hline Variables observables & 1 & 2 & 3 & 4 \\
\hline P7 Margen de mejora & 0.951 & & & \\
\hline P8 Adaptación tecnológica & 0.915 & & & \\
\hline P6 Alternativa de negocio & 0.913 & & & \\
\hline P10 Impactos económicos & 0.868 & & & \\
\hline P9 Tienda virtual & 0.865 & & & \\
\hline P38 Consecución de metas & & 0.887 & & \\
\hline P39 Organización y coordinación & & 0.854 & & \\
\hline P33 Integración de la ogranización & & 0.819 & & \\
\hline P36 Convicción de argumentos & & 0.736 & & \\
\hline P34 Capacidad de reinvención & & 0.729 & & \\
\hline P28 Invención & & & 0.826 & \\
\hline P26 Creatividad & & & 0.749 & \\
\hline P25 Nuevas ideas & & & 0.71 & \\
\hline P44 Cultura de innovación & & & 0.676 & \\
\hline P18 Orientación a consumidores & & & & 0.836 \\
\hline P21 Adaptabilidad a cambios & & & & 0.759 \\
\hline P22 Capacidad de resiliencia & & & & 0.684 \\
\hline
\end{tabular}

Fuente: Elaboración propia.

El Análisis Factorial Confirmatorio reduce de 45 items originales a 17 items en 4 constructos para estudiar el emprendimiento en las MiPymes en el sector comercial de Azogues, Ecuador: Valor para el cliente, P7 Margen de mejora, P8 Adaptación tecnológica, P6 Alternativa de negocio, P10 Impactos económicos y P9 Tienda virtual. Transformación digital, P38 Consecución de metas, P39 Organización y coordinación, P33 Integración de la organización, P36 Convicción de argumentos y P34 Capacidad de reinvención. Ventaja competitiva, P28 Invención, P26 Creatividad, P25 Nuevas ideas, P44 Cultura de innovación. Liderazgo, P18 Orientación a consumidores, P21 Adaptabilidad a cambios y P22 Capacidad de resiliencia (Veáse tabla 3).
Tabla 3 Constructo teóricos del emprendimiento

\begin{tabular}{|c|c|c|c|c|}
\hline \multirow[b]{2}{*}{ Variables observables } & \multicolumn{4}{|c|}{ Dimensiones } \\
\hline & \begin{tabular}{|l|} 
Valor \\
para el \\
cliente
\end{tabular} & $\begin{array}{l}\text { Transfor } \\
\text { mación } \\
\text { digital }\end{array}$ & $\begin{array}{l}\text { Ventaja } \\
\text { competi } \\
\text { tiva }\end{array}$ & Liderazgo \\
\hline P7 Margen de mejora & 0.951 & & & \\
\hline P8 Adaptación tecnológica & 0.915 & & & \\
\hline P6 Alternativa de negocio & 0.913 & & & \\
\hline P10 Impactos económicos & 0.868 & & & \\
\hline P9 Tienda virtual & 0.865 & & & \\
\hline P38 Consecución de metas & & 0.887 & & \\
\hline P39 Organización y coordinación & & 0.854 & & \\
\hline P33 Integración de la ogranización & & 0.819 & & \\
\hline P36 Convicción de argumentos & & 0.736 & & \\
\hline P34 Capacidad de reinvención & & 0.729 & & \\
\hline P28 Invención & & & 0.826 & \\
\hline P26 Creatividad & & & 0.749 & \\
\hline P25 Nuevas ideas & & & 0.71 & \\
\hline P44 Cultura de innovación & & & 0.676 & \\
\hline P18 Orientación a consumidores & & & & 0.836 \\
\hline P21 Adaptabilidad a cambios & & & & 0.759 \\
\hline P22 Capacidad de resiliencia & & & & 0.684 \\
\hline
\end{tabular}

Fuente: Elaboración propia

\section{CONCLUSIONES}

En este apartado se describen las conclusiones del estudio: El Análisis Factorial Confirmatorio aplicado a constructos del sector comercial en Azogues, Ecuador.

La investigación parte de una exhaustica inmersión en la literatura para identificar las dimensiones teóricas que se asocian al emprendimiento en las MiPymes del sector comercial del cantón Azogues. El rastreo realizado permite corroborar que el valor para el cliente, la transformación digital, la ventaja competitiva y el liderazgo se asociación con el emprendimiento y se convierten en dimensiones a ser probadas en el campo empírico.

Desde el punto de vista metodológico el Análisis Factorial Confirmatorio se convierte en una eficiente herramienta para el estudio del emprendimiento con un instrumento que contiene items con una carga factorial igual o 
superior 0.6 lo que determina la eliminación de coeficiente menores. De esta forma ser refina el instrumento de medición a un nivel de mayor precisión.

Una vez aplicado el Análisis Factorial Confirmatorio tomando como base los hallazgos de la literatura revisada se obtiene una importante reducción de items alrededor de 4 constructos teóricos que permiten estudiar el fenómeno de investigación y se puede responder con eficiencia a la pregunta central de investigación planteada.

El Análisis Factorial Confirmatorio constituye una herramienta que en el caso concreto del estudio ha permitido deshacerse de items que en vez de aportar por el contrario perjudican la confiabilidad del instrumento de medición para quedar solamente con aquellos items que aportan en gran medida a la precisión del análisis de los datos. 


\section{REFERENCIAS}

Aráuz, A. F. (2015). Aplicación del análisis factorial confirmatorio a un modelo de medición del rendimiento académico en lectura. Revista de Ciencias Económicas, 33(2), 39-65.

Arias, B. (2008). Desarrollo de un ejemplo de Análisis Factorial confirmatorio con LISREL, AMOS Y SAS. Publicaciones de INICIO, 75.

Anderson, J., Narus, J. A., \& Van Rossum, W. (2006). Propuestas de valor para el cliente en los mercados de empresas. Harvard Business Review, 86-97.

Banco Central del Ecuador (2021). Evolución de las Remesas. Quito, Ecuador.

Banco Central del Ecuador (2021). La economía ecuatoriana decreció en el segundo semestre del año 2020. Quito, Ecuador.

Buitrago, F. O. (2020). Revisión cronológica sobre liderazgo y emprendimiento. Interfaces, 2(2).

Cámara de Comercio de Guayaquil (2021). Informe Comercio propone: 40 Reformas al Código del Trabajo para la Creación de Empleos e impulso de la competitividad. Guayaquil, Ecuador.

De Frutos, B., Ruiz, M., \& San Martín, R. (1998). Análisis factorial confirmatorio de las dimensiones del compromiso con la organización. Psicológica, 345-366.

Domínguez, S. (2019). Correlación entre residuales en análisis factorial confirmatorio: una breve guía para su uso e interpretación. Interacciones, e207-e207.

Grupo de Investigación de Economía Regional (2020). Efectos que ocasionará el Covid-19 en la economía azuaya. Líderes.

Herrero, J. (2010). El Análisis Factorial Confirmatorio en el estudio de la Estructura y Estabilidad de los Instrumentos de Evaluación: Un ejemplo con el Cuestionario de Autoestima CA-14. Psychosocial Intervention, 19(3), 289-300.

Luna, A y Laca, F. (2014). Análisis Factorial Confirmatorio de la escala de satisfacción con la vida de familia (ESVF) en adolescentes de secundaria y bachillerato. Psicogente, 17(31), 226-240.

Magaña, D., Aguilar, N., \& Vázquez, J. (2017). Análisis Factorial Confirmatorio para medir las limitantes percibidas en el pregrado para el desarrollo de actividades de investigación. Revista Electrónica Nova Scientia, 515-536.

Morata, M; Holgado, F; Barbero, I y Méndez, G. (2015). Análisis Factorial Confirmatorio recomendaciones sobre mínimos cuadrados no ponderados en función del error tipo I de Jicuadrado y RMSEA. Acción Psicológica, 12(1), 79-90.

Pérez, D. O. (2020). Revisión del concepto de causalidad en el marco del Análisis Factorial Confirmatorio. Revista Iberoamericana de Diagnóstico y Evaluación-e Avaliação Psicológica, 1(54), 103-117.

Peñaloza Gómez, R (2019). El emprendimiento como ventaja competitiva.

Serna, M. D. A., John, W., Benavides, L. M. C., \& Burgos, D. (2018). Un modelo conceptual de transformación digital. Openergy y el caso de la Universidad Nacional de Colombia. Education in the Knowledge Society (EKS), 19(4), 95-107.

Sternberg, R; Prieto, M; \& Castejón, J. (2000). Análisis Factorial Confirmatorio del Sternberg Triarchic Abilities Test (nivel-H) en una muestra española: resultados preliminares. 
Suárez-Guerrero, C., Lloret-Catalá, C., \& Mengual-Andrés, S. (2016). Percepción docente sobre la transformación digital del aula a través de tabletas: un estudio en el contexto español. Comunicar: Revista Científica de Comunicación y Educación, 24(49), 81-89.

Valencia-Arias, A., Flórez, L. G., Restrepo, L. A. M., López, A. M. U. L. U., \& Restrepo, I. M. (2017). Consideraciones acerca del emprendimiento estratégico como síntesis funcional de la identificación de oportunidades y la creación de ventajas competitivas. Revista Virtual Universidad Católica del Norte, (52), 143-159.

Villamil, G. M. S. (2016). Liderazgo educativo en el siglo XXI, desde la perspectiva del emprendimiento sostenible. Revista Escuela de Administración de Negocios, (81), 111-128.

Viscarri, J. (2011). Modelo de creación de valor para el cliente. A: Congreso Internacional de Contaduría, Administración e Informática. "Memoria del XVI Congreso Internacional de Contaduría, Administración e Informática". México, DF: 2011, p. 1-17.

Zumbo, B. (2007. Análisis Factorial Confirmatorio recomendaciones sobre mínimos cuadrados no ponderados en función del error tipo I de Ji-cuadrado y RMSEA. Acción Psicológica. 